\title{
Inteins in mycobacterial GyrA are a taxonomic character
}

\author{
Peter Sander, ${ }^{1}$ Fernando Alcaide, ${ }^{2}$ Ingeborg Richter, ${ }^{1}$ Klaus Frischkorn, ${ }^{1}$ \\ Enrico Tortoli, ${ }^{3}$ Burkhard Springer, ${ }^{1}$ Amalio Telenti ${ }^{2}$ and Erik C. Böttger ${ }^{1}$
}

Author for correspondence: Peter Sander. Tel: +49511 532 4361. Fax: +495115324366.

e-mail: Sander.peter@mh-hannover.de

1 Institut für Medizinische Mikrobiologie,

Medizinische Hochschule

Hannover, Carl-Neuberg-

Str. 1, 30625 Hannover,

Germany

2 Département de Medicine Interne, Division des Maladies Infectieuses, CHUV-1011 Lausanne, Switzerland

3 Bacteriological and Virological Laboratory, Careggi Hospital, 50139 Firenze, Italy

\begin{abstract}
The A subunit of DNA gyrase in mycobacteria is frequently subjected to splicing events as its gene, gyrA, harbours an insertion encoding an intein. Investigation of a number of different isolates of Mycobacterium kansasii, Mycobacterium malmoense, Mycobacterium marinum, Mycobacterium ulcerans and Mycobacterium xenopi demonstrated that the presence of GyrA inteins is not random but a taxonomic character specific for a given taxon at a species or subspecies level.
\end{abstract}

Keywords: GyrA, intein, mycobacteria, Mycobacterium kansasii

\section{INTRODUCTION}

The term intein refers to unusual protein sequences that are excised from the precursor protein during maturation. Its coding sequence is always inserted in-frame with a protein-coding sequence (Perler et al., 1994). Protein maturation involves excision of the central protein from the precursor molecule and ligation of the $\mathrm{N}$ - and C-terminal domains to form the mature protein. Inteins probably possess endonuclease activity since they show homology to endonucleases of eukaryotes which mediate homing of the encoding sequence (Colston \& Davis, 1994).

Inteins have so far been described in the yeast vacuolar proton-pump ATPase subunit (Saccharomyces cerevisiae and Candida tropicalis) and in archaeobacterial DNA polymerases (Thermococcus litoralis and Pyrococcus spp.) (for a review see Colston \& Davis, 1994). Recently, as many as 18 putative inteins have been identified in the genome of Methanococcus jannaschii, an archaeon, from which the complete genome sequence has been determined (Bult et al., 1996).

In mycobacteria, inteins were first observed in the RecA protein of Mycobacterium tuberculosis and Mycobacterium leprae (Davis et al., 1992). More recently, it was found that inteins may be present in the A subunit of the mycobacterial DNA gyrase (Fsihi et al., 1996). The A subunit of the DNA gyrase, GyrA, is well-

The EMBL accession number for the sequence reported in this paper is AJ002066. conserved among bacteria and mediates double-strand breakage and reunion of DNA, while the B subunit is responsible for energy transduction via ATP hydrolysis (for review see Wigley, 1995). The intein coding sequences localize to the same position in $g y r A$ in different mycobacterial species, suggesting a specific homing site (Fsihi et al., 1996).

Among mycobacteria, the presence of GyrA inteins apparently is not uniform. While in gyrA of $M$. leprae intein coding sequences have been constantly observed, inteins have been found only occasionally in the corresponding proteins of Mycobacterium kansasii, Mycobacterium flavescens and Mycobacterium gordonae. Other mycobacterial species were investigated at a single strain level, thus excluding conclusions concerning the spreading of this characteristic feature (Fsihi et al., 1996). The finding that some strains of a given species have GyrA inteins, while others have not, prompted us to investigate the nature of this phenomenon.

\section{METHODS}

Strains. Several strains, isolated at Medizinische Hochschule Hannover (Germany), Zürich (Switzerland), Berne (Switzerland), Antwerpen (Belgium) and Firenze (Italy), each of Mycobacterium kansasii $(n=23)$, Mycobacterium malmoense $(n=8)$, Mycobacterium marinum $(n=8)$, Mycobacterium ulcerans $(n=4)$ and Mycobacterium xenopi $(n=$ 10) were included in this analysis.

Identification. Isolates were identified by conventional methods as well as by $16 \mathrm{~S}$ rRNA sequencing (Rogall et al., 1990). Strains of M. kansasii were also investigated by 
hybridization with a commercially available probe for $M$. kansasii (Gen-Probe; Tortoli et al., 1994a, b).

Molecular biological methods and protein alignment. PCR amplification of the non-coding 16S-23S rRNA intergenic spacer region was performed with primers 248 (5'-GTGTGGGTTTCCTTCCTTGG-3') and 42 (5'-CCACACGGGTTAACCTCGC-3') using standard conditions (Rogall $e t$ al., 1990). Nucleic acid sequencing was done manually using ${ }^{32} \mathrm{P}-$ labelled dCTP and sequenase (USB).

A previously devised PCR strategy using primers $\mathrm{H} 49$ (5'-AGGTTGTGCGGCGGGATATTGGT-3') and H50 (5'-TTCCGCCCGGACCGCAGCCACG-3') was used to investigate the presence of intein coding sequences in gyrA (Fsihi et al., 1996). Sequencing of gyrA intein coding sequence of M. malmoense was performed by PCR-mediated Taq cycle sequencing using an ABI373 sequencer. Protein alignment of known GyrA inteins (M. leprae, accession no. Z68206; $M$. flavescens, accession no. Z68209; M. gordonae, accession no. Z68208; M. kansasii, accession no. Z68207; and M. xenopi, accession no. U67876) was performed with the program CLUSTAL (PC/Gene, IntelliGenetics, release 6.85) as was the 16S rRNA sequence alignment of these species (Rogall et al., 1990).

\section{RESULTS AND DISCUSSION}

Mycobacteria with intein coding sequences in gyrA yield a PCR product of $1.6 \mathrm{kbp}$ or $0.9 \mathrm{kbp}$, whereas those with inteinless gyrA generate smaller fragments of around $350 \mathrm{bp}$.

Representative PCR results are presented in Fig. 1. The different isolates of $M$. malmoense, $M$. marinum, $M$. ulcerans and $M$. xenopi investigated showed a constant pattern: (i) isolates of M. malmoense were characterized by a gene fragment of $1.6 \mathrm{kbp}$, indicative of a GyrA intein; (ii) isolates of $M$. marinum and $M$. ulcerans showed an amplified gene fragment of $350 \mathrm{bp}$ charac- teristic for GyrA lacking an intein; (iii) isolates of $M$. xenopi presented a $0.9 \mathrm{kbp}$ fragment corresponding to a shorter intein coding sequence in $g y r A$, unique to this species. In contrast, isolates of $M$. kansasii were heterogeneous, producing either a $350 \mathrm{bp}$ or a $1.6 \mathrm{kbp}$ amplification fragment: 10 isolates were inteinless, while 13 isolates, including the $M$. kansasii type strain ATCC 12478 , were characterized by a $1.6 \mathrm{kbp}$ PCR product.

$M$. malmoense, $M$. marinum, $M$. ulcerans and $M$. xenopi are defined by a $16 \mathrm{~S}$ rRNA sequence which is identical for different isolates of a given species, while two distinct $16 \mathrm{~S}$ rRNA gene sequences have been reported for M. kansasii (Ross et al., 1992). Previously, the existence of two genetically distinct subspecies of $M$. kansasii has been suggested. These two subtypes can be differentiated by $16 \mathrm{~S}$ rRNA gene sequence determination (Ross et al., 1992), by hybridization to a probe derived from a repetitive DNA element (Yang et al., 1993b) or by hybridization to a commercially available probe for identification of M. kansasii (Gen-Probe; Yang et al., 1993a, b; Tortoli et al., 1994a). For the purpose of this study, these two groups of M. kansasii defined by $16 \mathrm{~S}$ rRNA and probe analysis are referred to as type I (GenProbe positive) and type II (Gen-Probe negative). The two different $16 \mathrm{~S}$ rRNA sequences found within our collection of $M$. kansasii isolates showed a complete correlation with the results of the probe assay.

Sequence analysis of the non-coding 16S-23S rRNA intergenic spacer region showed identical nucleic acid sequences for each of the different isolates of $M$. malmoense, M. marinum and M. ulcerans, while sequence heterogeneity was observed for the different isolates of $M$. kansasii (data not shown). This sequence heterogeneity matched the categorization by $16 \mathrm{~S}$ rRNA and genetic probe analysis. Thus, in contrast to $M$.

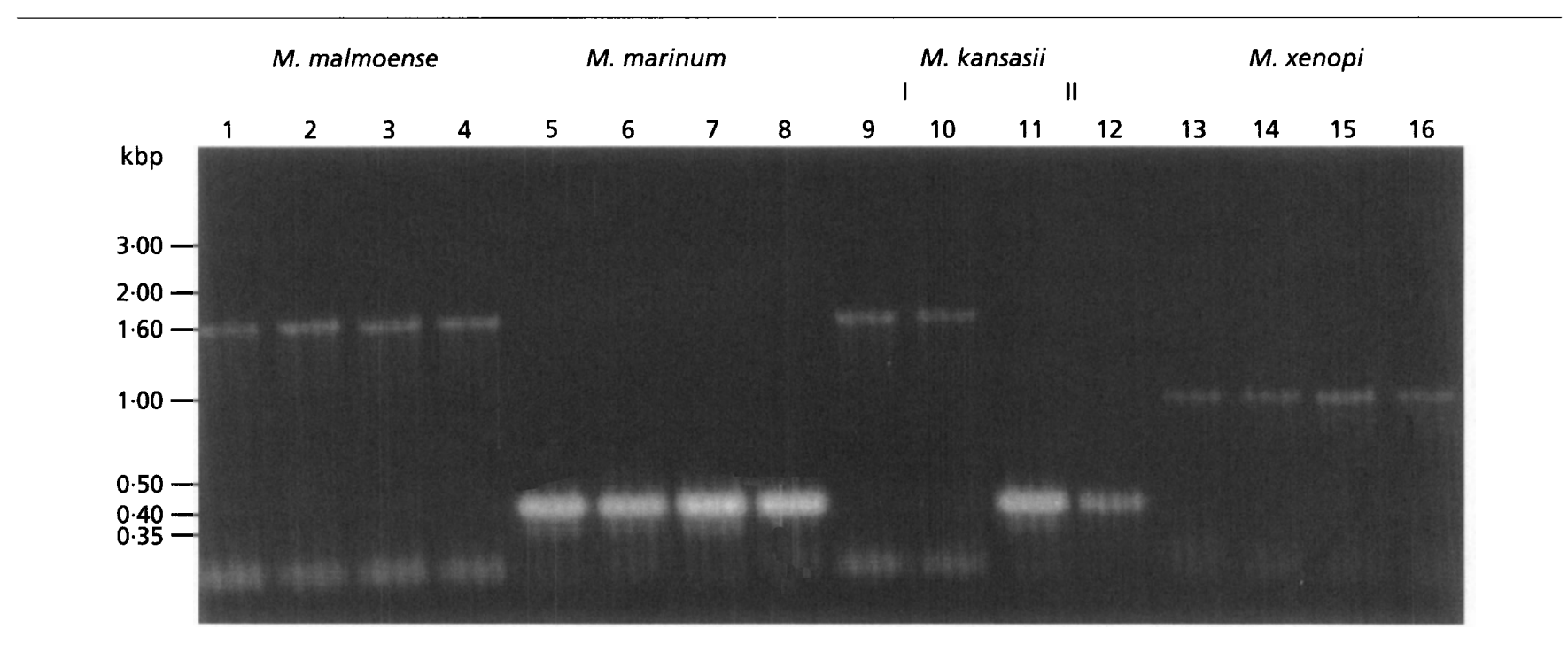

Fig. 1. PCR amplification of a gyrA gene fragment from different mycobacteria. Mycobacteria with intein coding sequences in gyrA yield a PCR product of $1.6 \mathrm{kbp}$ or $0.9 \mathrm{kbp}$, whereas those with inteinless GyrA generate a smaller fragment of around $350 \mathrm{bp}$. Lanes: $1-4, M$. malmoense; 5-8, M. marinum; 9 and 10, $M$. kansasii type $1 ; 11$ and $12, M$. kansasii type II; 13-16, M. xenopi. 
malmoense, M. marinum and M. ulcerans, which appear as genetically homogeneous taxons, M. kansasii genetically is more diverse and consists of two genetically distinct subspecies.

Analysis of the PCR amplification data (see Fig. 1) demonstrated that the presence of an intein coding sequence in gyrA was invariably associated with $M$. kansasii type I (13 of 13 investigated), whereas none of the type II $M$. kansasii isolates showed the presence of an intein coding sequence ( 10 of 10 investigated).

Sequence determination of PCR products obtained with primers $\mathrm{H} 49$ and $\mathrm{H} 50$ (Fsihi et al., 1996) and M. malmoense genomic DNA as template revealed an insertion following codon 130 of gyrA (Perler et al., 1997); the insertion comprises $1260 \mathrm{bp}$, encoding a putative intein of $420 \mathrm{aa}$. Comparison with gyrA genes of other mycobacterial species indicates a conservation of size ( 420 a in $M$. leprae, M. kansasii type I and $M$. gordonae and 421 aa in $M$. flavescens), the $\mathrm{N}$-terminal amino acid (Cys) and the C-terminal splice junction (His Asn/Thr). These GyrA inteins are much longer than the M. xenopi GyrA intein (198 aa), which lacks homing endonuclease activity and has undergone a complex series of recombination events (Telenti et al., 1997).

Phylogenetic trees based on 16S rRNA sequences indicate a closer relationship of $M$. malmoense, $M$. gordonae and $M$. kansasii to one another than to $M$. flavescens or $M$. leprae. A similar relationship was found when the GyrA intein sequences were compared (data not shown).

From our analysis, we draw the conclusion that the presence of a GyrA intein is not random but is a taxonomic character. This character defines a mycobacterial species either at a species or at a subspecies level depending on the degree of genetic homogeneity within a given taxon. Genetically homogeneous species such as $M$. malmoense, $M$. marinum, $M$. ulcerans, $M$. tuberculosis and $M$. leprae uniformly are characterized by the presence or absence of a GyrA intein. M. xenopi is characterized by its unique short gyrA element. In genetically more heterogeneous species, such as $M$. kansasii - and probably M. flavescens and M. gordonae (Fsihi et al., 1996) - the presence or absence of an intein coding sequence in gyrA corresponds to a subspecies characteristic. Our findings do not imply that the intein itself - although a valid taxonomic marker - is useful for defining phylogenetic relationships as the underlying mechanism of acquisition or loss of that marker remains to be determined.

\section{ACKNOWLEDGEMENTS}

We are indebted to Francoise Portaels and Gaby Pfyffer for providing strains. We thank Kerstin Teschner for excellent technical assistance.
This work was supported in part by grants from the Bundesministerium für Bildung, Wissenschaft, Forschung und Technik (Verbund Mykobakterielle Infektionen). E. C. B. is a Hermann- und Lilly-Schilling Professor of the Stifterverband für die Deutsche Wissenschaft. A. T. is supported by the Swiss National Science Foundation.

\section{REFERENCES}

Bult, J. C., White, O., Olsen, G. J. \& 37 other authors (1996). Complete genome sequence of the methanogenic archaeon, Methanococcus jannaschii. Science 273, 1058-1073.

Colston, M. J. \& Davis, E. O. (1994). The ins and outs of protein splicing elements. Mol Microbiol 12, 359-363.

Davis, E. O., Jenner, P. J., Brooks, P. C., Colston, M. J. \& Sedgwick, S. G. (1992). Protein splicing in the maturation of $M$. tuberculosis RecA protein: a mechanism for tolerating a novel class of intervening sequence. Cell 71, 201-210.

Fsihi, H., Vincent, V. \& Cole, S. T. (1996). Homing events in the gyrA gene of some mycobacteria. Proc Natl Acad Sci USA 93, 3410-3415.

Perler, F. B., Davis, E. O., Dean, G. E., Gimble, F. S., Jack, W. E., Neff, N., Noren, C. J., Thorner, J. \& Belfort, M. (1994). Protein splicing elements: inteins and exteins - a definition of terms and recommended nomenclature. Nucleic Acids Res 22, 1125-1127.

Perler, F. B., Olsen, G. J. \& Adam, E. (1997). Compilation and analysis of intein sequences. Nucleic Acids Res 25, 1087-1093.

Rogall, T., Flohr, T. \& Böttger, E. C. (1990). Differentiation of Mycobacterium species by direct sequencing of amplified DNA. J Gen Microbiol 136, 1915-1920.

Ross, B. C., Jackson, K., Yang, M., Sievers, A. \& Dwyer, B. (1992). Identification of a genetically distinct subspecies of Mycobacterium kansasii. J Clin Microbiol 30, 2930-2933.

Telenti, A., Southworth, M., Alcaide, F., Daugelat, S., Jacobs, W. R. \& Perler, F. B. (1997). The Mycobacterium xenopi GyrA protein splicing element: characterization of a minimal intein. J Bacteriol 179, 6378-6382.

Tortoli, E., Simonetti, M. T., Lacchini, C., Penati, V., Piersimoni, C. \& Morbiducci, V. (1994a). Evaluation of a commercial DNA probe assay for the identification of Mycobacterium kansasii. Eur J Clin Microbiol Infect Dis 13, 264-267.

Tortoli, E., Simonetti, M. T., Lacchini, C., Penati, V. \& Urbano, P. (1994b). Tentative evidence of AIDS-associated biotype of Mycobacterium kansasii. J Clin Microbiol 32, 1779-1782.

Wigley, D. B. (1995). Structure and mechanism of DNA topoisomerases. Annu Rev Biophys Biomol Struct 24, 185-208.

Yang, M., Ross, B. C. \& Dwyer, B. (1993a). Identification of an insertion sequence-like element in a subspecies of Mycobacterium kansasii. J Clin Microbiol 31, 2074-2079.

Yang, M., Ross, B. C. \& Dwyer, B. (1993b). Isolation of a DNA probe for identification of Mycobacterium kansasii, including the genetic subgroup. J Clin Microbiol 31, 2769-2772.

Received 1 April 1997; revised 23 September 1997; accepted 1 October 1997 